\title{
Piling and avalanches of magnetized particles
}

\author{
S. Fazekas ${ }^{1,2}$, J. Kertész ${ }^{1}$, and D. E. Wolf ${ }^{3}$ \\ ${ }^{1}$ Department of Theoretical Physics, \\ Budapest University of Technology and Economics, \\ H-1111 Budapest, Hungary \\ ${ }^{2}$ Theoretical Solid State Research Group of the Hungarian Academy of Sciences, \\ Budapest University of Technology and Economics, \\ H-1111 Budapest, Hungary \\ ${ }^{3}$ Institute of Physics, University Duisburg-Essen, \\ 47048 Duisburg, Germany
}

(Dated: April 7, 2004)

\begin{abstract}
We performed computer simulations based on a two-dimensional Distinct Element Method to study granular systems of magnetized spherical particles. We measured the angle of repose and the surface roughness of particle piles, and we studied the effect of magnetization on avalanching. We report linear dependence of both angle of repose and surface roughness on the ratio $f$ of the magnetic dipole interaction and the gravitational force (interparticle force ratio). There is a difference in avalanche formation at small and at large interparticle force ratios. The transition is at $f_{c} \approx 7$. For $f<f_{c}$ the particles forming the avalanches leave the system in a quasi-continuous granular flow (granular regime), while for $f>f_{c}$ the avalanches are formed by long particle clusters (correlated regime). The transition is not sharp. We give plausible estimates for $f_{c}$ based on stability criteria.
\end{abstract}

PACS numbers: 45.70.-n, 45.70.Cc, 45.70.Ht

Keywords: granular systems, static sandpiles, avalanches

\section{INTRODUCTION}

The dipole interaction between magnetized particles can be viewed as an anisotropic adhesion force. Because its strength can be easily manipulated by the strength of the magnetizing field, magnetized particles have been recently proposed [1, 2] to get insight into the transition from noncohesive to cohesive grains. Previously adhesion effects have mainly been studied in form of moistureinduced changes, significant for industrial processes in fields as pharmaceuticals, agriculture, and constructions.

Some time ago Hornbaker et al. 3] addressed the question how sand castles stand. They stated that already small quantities of wetting liquid can dramatically change the properties of granular media, leading to large increase in the angle of repose and correlation in grain motion. Theoretical studies on the angle of repose based on stability criteria have been done by Albert et al. [4] They theoretically determined the dependence of the angle of repose on cohesive forces, and applied the results to wet granular material.

Experimental studies of Tegzes et al. on angle of repose using the draining crater method [5] and on avalanches using a rotating drum apparatus 6] identify three distinct regimes as the liquid content is increased: a granular regime in which the grains move individually, a correlated regime in which the grains move in correlated clusters, and a plastic regime in which the grains flow coherently.

Experiments of Quintanilla et al. 7] using the rotating drum apparatus address the question of self-organized critical behavior in avalanches of slightly cohesive powders. Their results show that avalanche sizes do not follow a power-law distribution, however, they scale with powder cohesiveness. Samadani et al. [8] studied the effect of interstitial fluid on the angle of repose and the segregation of granular matter poured into a quasi-twodimensional silo.

To study the transition from noncohesive to cohesive behavior, Forsyth et al. [1, 2], adopting the widely suggested idea that competition between the interparticle forces and the inertial forces determines the behavior of cohesive granular materials, suggested a method based on magnetized particles. The particles placed in an external magnetic field become magnetized, all having the same magnetic orientation parallel to the field. Varying the strength of the field allows to continuously vary the resulting interparticle magnetic force. Using nonmagnetic perspex walls the particle-wall interaction remains unchanged relative to the noncohesive state. Using particles under same packing conditions it is ensured that the initial conditions are as uniform as possible.

We carried out computer simulations on a system corresponding to the experiments of Forsyth et al. [1, 2] and studied the angle of repose, the surface roughness, and the effect of magnetization on avalanching in twodimensional particle piles.

The magnetic interaction of magnetized grains is highly anisotropic, and the fixed external field introduces even more anisotropy as the grains are aligned to the field. A similar experimental setup [9], but with particles carrying a remanent magnetization in the absence of an external magnetic field, would partly diminish the mentioned anisotropy, however in this case the magnetizations and the interparticle forces are not as well defined as in the experiments of Forsyth et al. [1, 2].

Because of the strong anisotropy and the longer interaction range one can expect differences between the results on magnetized particles and wet granular systems, 
however the basic effects should be the same.

\section{SIMULATION METHOD}

We performed computer simulations based on a twodimensional Distinct Element Method (DEM) [10] (for a review see [11, 12, 13] and references therein) to study granular systems of magnetized spherical particles. The particles are magnetized by a constant external field, all having the same magnetic orientation parallel to the field. The magnetization is modeled with dipoles. We neglect any coupling between the magnetic orientation and particle rotation (i.e., the particles can rotate freely, while their magnetic dipole is fixed).

For characterizing the strength of the interparticle force, we introduce a dimensionless quantity defined by the ratio of the maximum magnetic interparticle force at contact and the gravitational force.

The magnetic force acting on a dipole $\mathbf{m}_{2}$ situated at distance $r_{21}$ from a dipole $\mathbf{m}_{1}$, along the direction $\mathbf{n}_{21}$ is given by

$$
\begin{array}{r}
\mathbf{F}_{21}=\frac{\mu_{0}}{4 \pi} \frac{3}{r_{21}^{4}}\left[\left(\mathbf{n}_{21} \mathbf{m}_{2}\right) \mathbf{m}_{1}+\left(\mathbf{n}_{21} \mathbf{m}_{1}\right) \mathbf{m}_{2}\right. \\
\left.-5\left(\mathbf{n}_{21} \mathbf{m}_{1}\right)\left(\mathbf{n}_{21} \mathbf{m}_{2}\right) \mathbf{n}_{21}+\left(\mathbf{m}_{1} \mathbf{m}_{2}\right) \mathbf{n}_{21}\right] .
\end{array}
$$

For identical hard spherical particles of diameter $D$ and magnetic dipole $S$, the largest possible dipole-dipole magnetic force is

$$
F_{m}=\frac{\mu_{0}}{4 \pi} \frac{6 S^{2}}{D^{4}}
$$

which corresponds to a head-to-tail configuration, the dipoles having the same orientation.

We define the magnetic interparticle force ratio as

$$
f=F_{m} / F_{g},
$$

where $F_{g}=m g$ is the gravitational force ( $m$ denotes the particle mass and $g$ is the gravitational acceleration).

Considering mass density $\rho$ and magnetization $M$, we have $m=\rho V, S=M V, V=\pi D^{3} / 6$, and thus

$$
f=\frac{F_{m}}{F_{g}}=\frac{\mu_{0}}{4 \pi} \frac{6 S^{2}}{m g D^{4}}=\frac{\mu_{0}}{4 \pi} \frac{\pi M^{2}}{\rho g D} .
$$

Assuming some $f$ interparticle force ratio, from the previous equation the corresponding magnetization can be calculated as

$$
M=\left(f \frac{4 \pi}{\mu_{0}} \frac{\rho g D}{\pi}\right)^{1 / 2} .
$$

In our simulations we used $\rho=7.5 \mathrm{~g} / \mathrm{cm}^{3}$ (which corresponds approximately to the mass density of steel), $g=9.8 \mathrm{~m} / \mathrm{s}^{2}$, and interparticle force ratio $f<25$.

The diameter of the spherical particles was taken from the $0.7-0.9 \mathrm{~mm}$ interval, with a Gauss-like distribution having the mean of $0.8 \mathrm{~mm}$. The vast majority of particles had diameters very close to $D=0.8 \mathrm{~mm}$. The Gauss-like distribution is given by the average of 4 independent uniformly distributed random variables in the mentioned interval. This slight polydispersity, resembling real experimental setups, is used to avoid effects originated from symmetries of monodisperse systems.

The long range magnetic interaction is taken in consideration within a reasonable cutoff distance as a dipoledipole interaction. We choose the magnetic interaction cutoff to $6.25 D$ (where $D$ is the average particle diameter). As shown in a previous study [14], $5 D$ value already gives a reasonable magnetic interaction cutoff in twodimensional dipolar hard sphere systems regarding the local ordering. The angle of repose, the surface roughness, and the particle avalanches depend crucially on local orderings inside the pile, as noted for example by Altshuler et al. in [15]. The used cutoff keeps the character of local orderings and changes the magnetic energy per particle by less than $5 \%$ 14].

We calculate the collision interaction of particles using the Hertz contact model [16] with appropriate damping 17]. We implement Coulomb sliding friction for large relative translational velocities and for numerical stability viscous friction for small velocities, with continuous transition between the two, controlled by a (large) viscous friction coefficient. We do not use any static or rolling friction model. A grid based method is used to identify neighboring (and potentially colliding) particle pairs.

The parameters of the Hertz contact model were chosen such that they correspond to Young modulus of approximately $0.015 \mathrm{GPa}$ and restitution coefficient of approximately 0.86 . These are characteristic values for hard rubber elastomers (used for example in constructing golf ball covers). The Young modulus is orders of magnitudes smaller than that of steel, a choice enabling realistic CPU times with the DEM method. The particle-particle and the particle-wall sliding friction coefficient was 0.5 and 0.7 (characteristic for steel-steel and steel-perspex friction).

The translational motion of particles is integrated based on Newton's equation using Verlet's leap-frog method. The rotational state of particles is integrated with Euler's method. The integration time step was $5 \mu s$. With the used elastic parameters a good lower estimate for collision times is $170 \mu \mathrm{s}$. In such conditions, the used integration time step gave good numerical stability and also fairly good response time on PCs with $1.8 \mathrm{GHz}$ CPUs available at the time of writing.

The simulation setup can be seen in Fig. 1 The external magnetic field is vertical. The particles are added one by one with constant rate along vertical trajectories at small (maximum one particle diameter) random dis- 


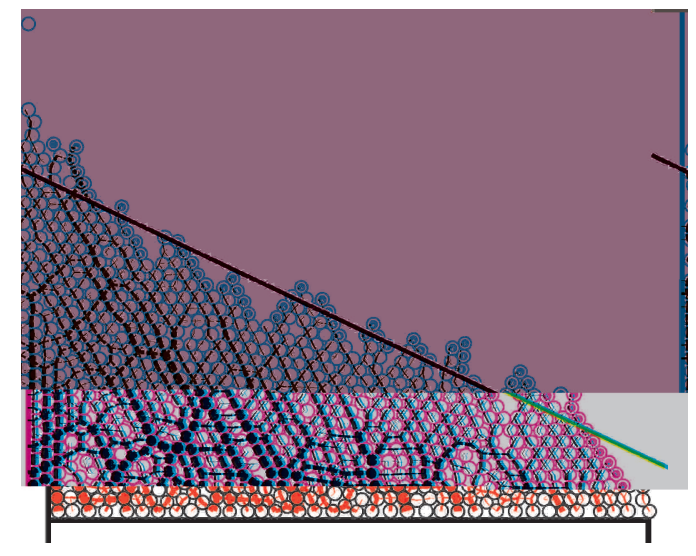

FIG. 1: Simulation setup. The particles are introduced with constant rate one by one at small random distances from the left wall. The particles can leave the system on the right side. The surface angle (i.e. the angle of repose) is measured by fitting a straight line over the positions of the surface particles (marked with black). The figure also shows the normal contact forces. The thickness of the lines connecting the centers of the particles in contact is proportional to the normal contact force. The sample corresponds to $f=6$ interparticle force ratio. For color figure see the electronic version.

tance from the left wall. They either reach the pile with a given velocity (i.e. they are fired into the pile), or their impact velocity is set to zero (i.e. they are placed gently on the pile).

The system's bottom wall is sticky. Any particle touching the bottom wall sticks to the wall. This builds up a random base (see the experimental setup used by Altshuler et al. described in [15]). The particles can leave the system on the right side. The particles are removed from the simulation when their distance from the bottomright corner is larger than the magnetic interaction cutoff distance. The system's bottom is $51.25 \mathrm{D}$ length. Only this system size was used, finite size effects were not studied.

The surface particles (marked with black in Fig. 1) are identified with the weighted alpha shape algorithm [18, 19]. Alpha shapes are generalizations of the convex hull and can be used for shape reconstruction from a dense unorganized set of data points. The weighted alpha shapes are extensions of this kind of shape reconstruction to a set of spheres (as in our case). We used the implementation included in the Computational Geometry Algorithms Library [20]. The algorithm's alpha parameter was set to the square of the mean particle size. This gave satisfactory results. The surface angle (i.e. the angle of repose) is measured by fitting a straight line over the positions of the surface particles. The surface roughness is given by the standard deviation of the surface points from the fitted line.

As part of our investigations, with a special side wall model, we also simulated the effect of front and back walls in a Hele-Shaw cell geometry encountered in experimen- tal studies. We took into consideration the frictional interaction with side walls by summing the magnitude of normal forces acting on one particle, directing a well defined percentage of this pressure on the walls, and deriving a frictional force using the already mentioned friction model. The percentage of the total force directed on the side walls was a parameter of our simulations.

We performed three sets of simulations: (a) the particles were fired into the pile, (b) the particles were placed gently on the pile, and (c) the particles were fired into the pile, while $4 \%$ of the internal pressure was directed on the front and back walls (see side wall model). In both (a) and (c) the particles reached the pile with $0.5 \mathrm{~m} / \mathrm{s} \mathrm{im-}$ pact velocity, which corresponds to approximately $16 \mathrm{D}$ dropping height. In all three simulation sets we executed runs at different interparticle force ratios. In each run we started with an empty system, and introducing 12000 particles, one particle every 3000 integration steps, we numerically integrated the system for 3 minutes (simulated time).

In the first part of the process the number of particles in the system increased monotonically. After a pile was built, avalanches started, which in a pulsating manner moved particles out of the system. In this way the number of particles began to oscillate around some well defined value. In this latter part we identified the surface particles every 500 integration steps, and we measured the slope of the fitted surface line and the standard deviation of surface points from this line. The average of this quantities over the simulated time gave the measured angle of repose and surface roughness.

We also measured the avalanche sizes and avalanche durations. This can be done in many different ways. We define an avalanche by a number of individual events on the time scale of the integration steps (i.e. the smallest simulation time step) in which (at least) one particle leaves the system, and the time between two consecutive events is smaller than a well defined value. We take this value equal to the time corresponding to 3000 integration time steps. Our choice is based on the system's observed dynamic time scale, and the fact that one new particle is introduced (i.e. the system is perturbed) every 3000 integration time steps, and thus on a larger time scale there are surely uncorrelated events.

\section{SIMULATION RESULTS}

\section{A. Angle of repose and surface roughness}

In all cases both the angle of repose and the surface roughness (in the examined domain) exhibit a linear dependence on the interparticle force ratio (see Fig. 21).

The angle of repose in case (a) and (b) increases by approximately 0.5 degree per unit change of interparticle force ratio (see upper panel in Fig. 2). This is in good accordance with the experimental results of Forsyth et al. [1, 2], however the angle of repose at zero magnetization 

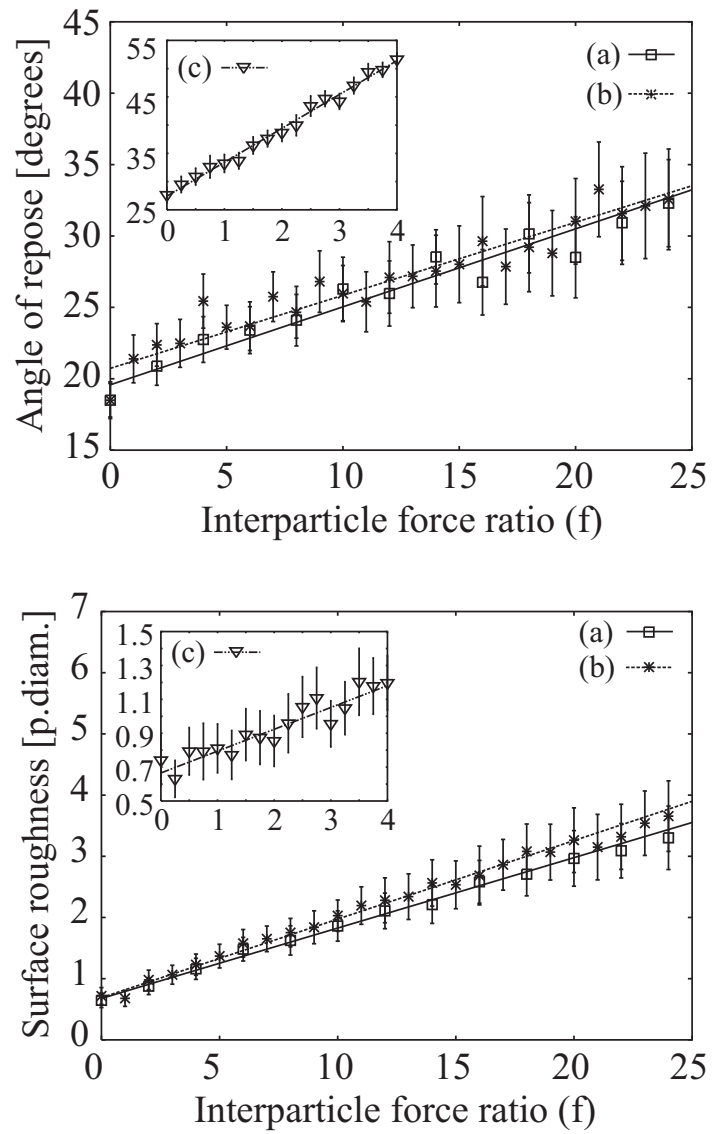

FIG. 2: Angle of repose (upper panel) and surface roughness (lower panel) at different magnetic interparticle force ratios. The angle of repose is measured in degrees. The surface roughness is measured in (average) particle diameters. We executed three set of simulations. In (a) and (c) the particles were fired into the pile, while in (b) the particles were placed gently on the pile. In (c) an artificial side wall effect was switched on (see text for details).

in our case is about 10 degrees smaller. This can be the result of the missing side wall effect (see for example [21]), and the missing static and rolling friction (see for example 22, 23]).

At zero magnetization the average surface roughness is about 0.7 particle diameters, and in all cases increases by approximately 0.12 particle diameters per unit change of interparticle force ratio (see lower panel in Fig. 2).

As a consequence of our side wall model, the angle of repose in case (c) is about 8 degrees higher at zero magnetization in agreement with experimentally observed effects of front and back walls in Hele-Shaw cells. However, the way we model the side walls leads to a stronger increase of the angle of repose with $f$ than in the experiments of Forsyth et al. 1, 2] (see inset of upper panel in Fig. 2). The side wall effect does not influence the surface roughness (see inset of lower panel in Fig. 21).
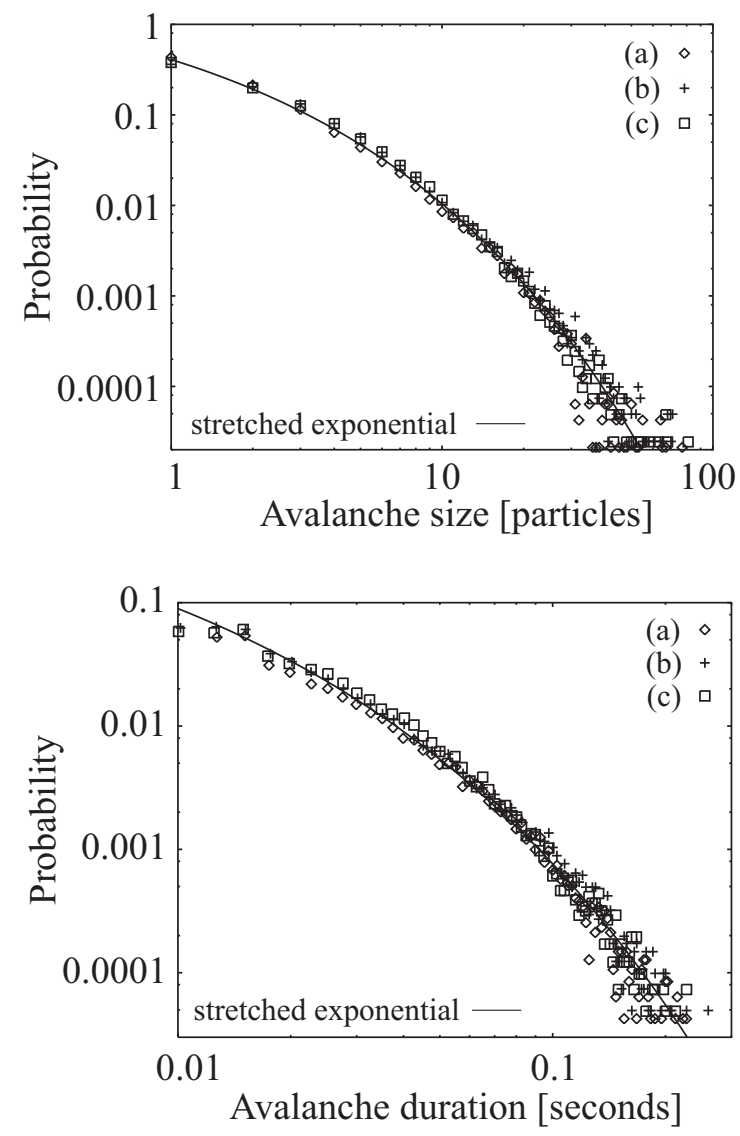

FIG. 3: Distribution of particle avalanche sizes (upper panel) and avalanche durations (lower panel) at zero magnetization. We examined three different simulation setups (see text for details). Firing the particles into the pile (i.e. dropping them from a given height) or placing them gently, and switching on or off the side wall effect gave no qualitative difference. Over our simulation data (for both avalanche sizes and avalanche durations) we could fit a stretched exponential with $\gamma=0.43$.

\section{B. Particle avalanches at zero magnetization}

We carefully examined the distribution of particle avalanches at zero magnetization in all three simulation sets. We also executed extra runs introducing a total of 144000 particles, one particle every 3000 integration steps, integrating for a total of 36 minutes (simulated time). It must be noted that simulating at zero magnetization (i.e. without magnetization) is about 10 times faster, because only the (short-range) collision interaction must be calculated. This permitted longer simulation times. The corresponding particle avalanche size and duration statistics can be seen in Fig. 3

Firing the particles into the pile (i.e. dropping them from a given height) or placing them gently, and switching on or off the side wall effect gave no qualitative difference. Over both the avalanche size and duration distribution data we could fit stretched exponentials of form 


$$
P(x)=P_{0} \exp \left[-\left(x / x_{0}\right)^{\gamma}\right]
$$

with $\gamma=0.43$.

Our $\gamma$ value is in good agreement with the experimental results of 24 and is the same value as the one found by Frette et al. 25] for piles of rice with small anisotropy. By contrast recent work of Costello et al. [26] presents carefully collected and detailed experimental results on piling of uniform spherical glass beads, which show a power law behavior with an exponential cutoff. Costello et al. argue that the exponential cutoff depends on the height from which the particles are dropped and probably also on cohesion forces. It was not our intention in this study to contribute to the clarification of this point.

\section{Effect of magnetization on particle avalanching}

We analyzed the effect of magnetization on particle avalanching, and we found that there is a difference in avalanche formation at small and at large interparticle force ratios (see avalanche movies [27]). We identified a granular and a correlated regime. The transition between the two regimes is not sharp. Similar regimes were identified experimentally by Tegzes et al. [5, 6] in case of wet granular materials. At high liquid content (i.e. large interparticle force ratio) they could also identify a third plastic regime.

Studying the recordings from our simulations 27, it can be observed that for small magnetizations the avalanches are formed by small vertical chains following each other at short times, giving the impression of a quasi-continuous flow (granular regime). As the particle magnetization increases, at $f_{c} \approx 7$ the previously quasi-continuous flow is replaced by individual narrow and long particle clusters falling at the system's boundary (correlated regime).

There are two elementary processes characteristic for the dynamics of avalanche formation: peeling and splitting (see Fig. (4). Near the foot of the pile the particles are arranged approximately in a triangular lattice with a horizontal base. By contrast magnetic interactions would favor a triangular lattice with a vertical base. Clusters of particles near the free surface of the pile can peel off the pile by rotating 30 degrees into this favorable configuration (see part (i) of Fig. (4). A favorably oriented domain of particles on a triangular lattice consists of vertical chains shifted by half a particle diameter from one chain to the next. This domain may be stable or may disintegrate into smaller domains or chains, depending on whether or not the magnetic interactions are strong enough to prevent the dilation, which is necessary to allow for relative motion of chains within the domain (see part (ii) of Fig. (4).

Combinations of these two processes determine the size of the outflowing clusters, which typically consist of $\nu$

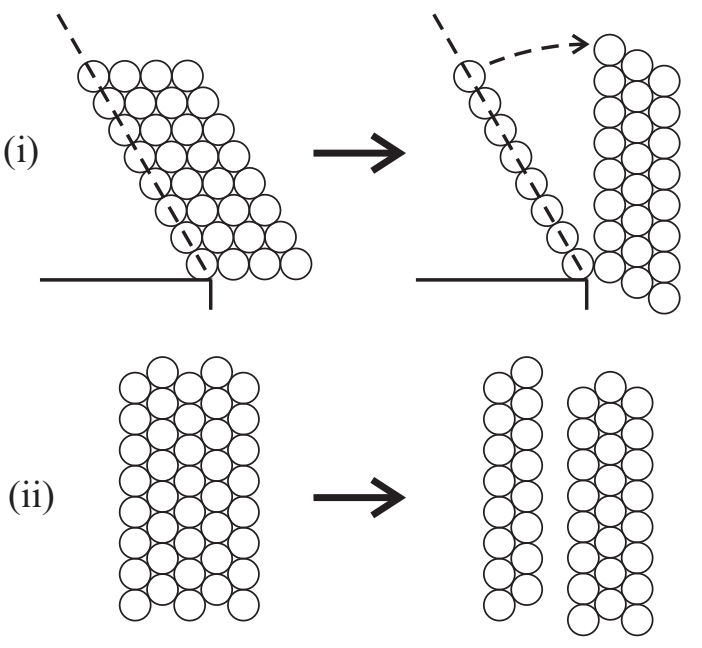

FIG. 4: Elementary processes characteristic for the dynamics of avalanche formation: (i) peeling and (ii) splitting. A particle cluster composed of parallel chains can peel off the pile by rotating to a more favorable orientation, and can disintegrate into clusters composed of smaller number of chains. Combinations of these two processes determine the size of the outflowing clusters.

parallel vertical chains of length $N$. Both $N$ and $\nu$ depend on $f$. Taking into account only the magnetic interaction between nearest neighbors one can give plausible estimates for this dependence.

We compare the gain in magnetic energy and the loss in gravitational energy when a cluster of particle chains of length $N$ rotates by an angle $\alpha$ into a position where the chains are aligned with the magnetic field. The magnetic moment of each particle is always aligned with the magnetic field, so that only the dipolar interaction between the grains matters. We take into consideration only intrachain interactions, neglecting the interchain interactions. The dipole-dipole-interaction potential between two particles whose center of mass connection is tilted by an angle $\alpha$ with respect to the magnetic field is

$$
E_{m}=\frac{\mu_{0}}{4 \pi} \frac{S^{2}}{D^{3}}\left(1-3 \cos ^{2} \alpha\right) .
$$

Hence rotating them into the field direction $(\alpha=0)$ lowers the energy per particle in the cluster by an amount proportional to

$$
\Delta E_{m}=\frac{\mu_{0}}{4 \pi} \frac{3 S^{2}}{D^{3}}\left(1-\cos ^{2} \alpha\right) .
$$

The center of mass is lifted by this rotation. Therefore the gravitational potential energy per particle in the cluster increases by

$$
\Delta E_{g}=N \frac{m g D}{2}(1-\cos \alpha) .
$$


Setting $\alpha=30$ degree and using the definition of $f$ given in Eq. (4), $\Delta E_{m}=\Delta E_{g}$ shows that clusters up to chain length

$$
N_{\max } \approx(1+\sqrt{3} / 2) f
$$

can peel off.

In order to check to what thickness $\nu_{\max }$ such a cluster is stable with respect to splitting into subclusters with less chains, we compare the magnetic energy loss per unit chain length with the gravitational energy gained, when a subcluster of $\nu$ chains moves down by half a particle diameter relative to the rest of the cluster. In this case we have

$$
\Delta E_{m}=\frac{\mu_{0}}{4 \pi} \frac{S^{2}}{2 D^{3}}, \text { and } \Delta E_{g}=\frac{m g D}{2} \nu .
$$

This shows that the dipole-dipole-interaction can only prevent cluster splitting, if the cluster consists of less than

$$
\nu_{\max } \approx \frac{1}{6} f
$$

chains.

Based on the above results we can discuss the process of avalanche formation. Already at small magnetizations the surface roughness allows for coherent rotation of larger clusters up to chain length $N_{\max }$. For $f<f_{c} \approx 6$ clusters consisting of more than one chain of particles can easily dilate and will disintegrate into isolated chains, forming a quasi-continuous flow. For $f>f_{c}$ clusters consisting of up to $\nu_{\max }>1$ chains can fall. These results are close to the observations made on the simulations: The transition between the two regimes was observed at $f_{c} \approx 7$.

The difference in avalanche formation in different regimes can be clearly observed in avalanche duration to avalanche size relation. In all three simulation sets, at given interparticle force ratios, for each avalanche size we collected the measured avalanche durations and calculated the corresponding average avalanche durations. We also examined the avalanche size and duration distributions. Our results are summarized in the next two subsections.

\section{The granular regime}

In the granular regime the avalanche sizes are proportional to the corresponding average avalanche durations. The proportionality factor (i.e. the ratio of avalanche sizes and average avalanche durations) defines an average avalanche flow, which increases linearly with $f$ (see Fig. (5). This linear dependence is explained by the fact that the avalanches in granular regime are formed by

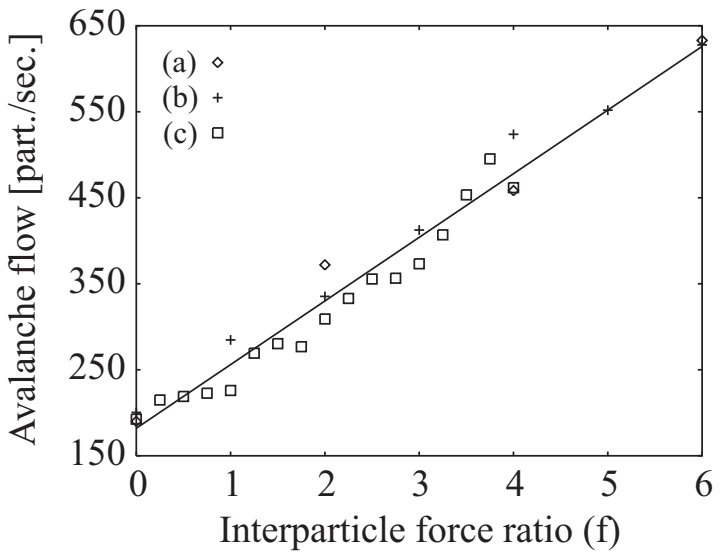

FIG. 5: Dependence of average avalanche flow on interparticle force ratio in granular regime. We examined three different simulation setups (see text for details). Firing the particles into the pile (i.e. dropping them from a given height) or placing them gently, and switching on or off the side wall effect gave no qualitative difference. The avalanche flow is measured in particles per second.

quasi-continuous flows of small particle chains, and the height of these chains increases linearly with $f$.

Independent of the setup, at zero magnetization the average avalanche flow is approximately 182 particles per second, and increases with approximately 74 particles per second per unit change of interparticle force ratio (see Fig. (5).

The avalanche size distribution at interparticle force ratios $1<f<7$ can be scaled together reasonably well (see Fig. [6) using the ansatz

$$
P(s, f)=f^{-1} Q(s / f)
$$

where $s$ denotes avalanche sizes, $P(s, f)$ is the probability associated with an avalanche size, and $Q(\cdot)$ is a function with integral 1 on the $[0,+\infty)$ interval.

Based on the avalanche size distributions (see Fig. 6), we argue that the magnetic cohesion introduces a welldefined characteristic size in particle avalanches. From the scaling property, we conclude that, the characteristic avalanche size increases linearly with the interparticle force ratio. Qualitatively similar results were found in the experiments by Szalmás et al. [9].

As both the characteristic avalanche size and the average avalanche flow increase linearly with $f$, the characteristic average avalanche duration (equal to the ratio of the former two) in leading order is independent of $f$. The dependence of the avalanche duration distribution on $f$ is contained in higher order corrections which could not be captured by our simulations. 


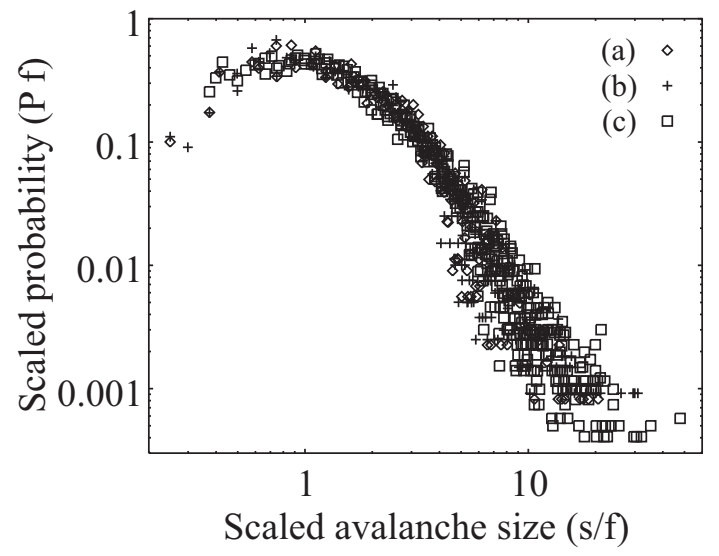

FIG. 6: Scaled avalanche size distribution in granular regime. A well-defined characteristic size can be observed. We examined three different simulation setups (see text for details). The avalanche size distribution at interparticle force ratios $1<f<7$ are scaled together using the ansatz $P(s, f)=$ $f^{-1} Q(s / f)$, where $s$ denotes avalanche sizes. From the scaling property, we conclude that, the characteristic avalanche size increases linearly with $f$.

\section{E. The correlated regime}

In correlated regime the avalanche durations are given by the free fall of long particle clusters, and accordingly the square of the measured avalanche durations are proportional to the length of the clusters, and thus proportional to $f$. At the same time, as it was already mentioned, the width of the falling particle clusters is small, and thus the avalanche sizes in leading order are proportional to the cluster length. In consequence the avalanche sizes are proportional to the square of the corresponding average avalanche durations, contrary to the linear dependence found in granular regime.

As it was already mentioned, the width of the falling particle clusters can take arbitrary values up to some well defined maximum. In other words, the particle clusters can have different number of layers while having the same length. This introduces large fluctuations in avalanche sizes. These fluctuations are proportional to the cluster length, and thus proportional to $f$.

The avalanche duration distributions at interparticle force ratios $7<f<25$ can be scaled together reasonably well (see Fig. 17) using the ansatz

$$
P(\tau, f)=f^{-1 / 2} Q\left(\tau / f^{1 / 2}\right),
$$

where $\tau$ denotes avalanche durations, $P(\tau, f)$ is the probability associated with an avalanche duration, and $Q(\cdot)$ is a function with integral 1 on the $[0,+\infty)$ interval.

A well-defined characteristic duration can be observed in Fig. [7 Based on the scaling property, the square of the characteristic avalanche duration increases linearly with the interparticle force ratio. As the square of the

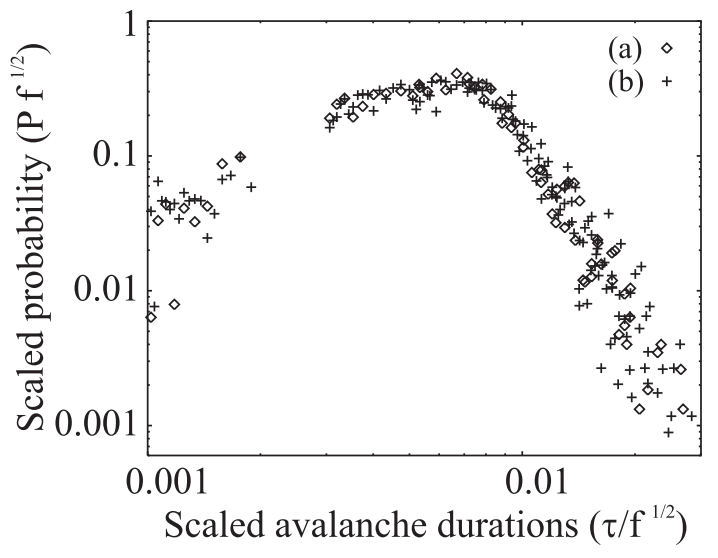

FIG. 7: Scaled avalanche duration distribution in correlated regime. A well-defined characteristic duration can be observed. We examined two simulation setups (see text for details). The avalanche duration distribution at interparticle force ratios $7<f<25$ are scaled together using the ansatz $P(\tau, f)=f^{-1 / 2} Q\left(\tau / f^{1 / 2}\right)$, where $\tau$ denotes avalanche durations. From the scaling property, we conclude that, the square of characteristic avalanche duration increases linearly with $f$.

avalanche duration is proportional to a corresponding cluster length, and a cluster length in leading order defines an avalanche size, we can conclude that there is also a mean characteristic avalanche size, which consequently is proportional to $f$. We could not find this avalanche size explicitly in the avalanche size distribution because of the large fluctuations of the same order of magnitude as the mean value.

\section{CONCLUSIONS AND DISCUSSION}

We studied basic effects in two-dimensional granular piles formed by magnetized particles in simulations similar to the experiments of Forsyth et al. 1, 2]. We measured the angle of repose, the surface roughness, and the effect of magnetization on particle avalanching. As a general result we can mention that dropping the particles from a given height or placing them gently, and switching on or off the effect of the side walls gave no qualitative difference.

We found that both the angle of repose and the surface roughness exhibits linear dependence on the ratio $f$ of the maximum magnetic force at contact and the gravitational force. As it was also mentioned by Forsyth et al. [1, 2], $f$ overestimates the effective cohesion because of the anisotropy of the magnetic interaction. According to this, the angle of repose increases much more slowly with $f$ than expected from stability criteria [4] and experiments [5] on wet granular media. The experimental results of Forsyth et al. and our simulations are in good accordance, though the angle of repose at zero magnetization in our case was smaller. The side wall model taking 
into consideration the effect of the front and back walls of a Hele-Shaw cell arrangement, could increase the surface angle but did also introduce a stronger dependence of the angle of repose on $f$ than in the experiments of Forsyth et al. 1, 2]. Taking into consideration the static and rolling friction of particles could probably reproduce more closely the experimental results in this respect too.

Tegzes et al. 5] found in experimental studies of wet granular media linear dependence of the angle of repose on the interparticle force ratio in the granular regime and an almost linear dependence in correlated regime with a slight curvature. This bending could not be clearly identified in our results and most probably would require more accurate investigations at both small and large interparticle force ratios.

As reported by Tegzes et al. [6] in case of wet granular media there is a difference in avalanche formation at small and at large interparticle force ratios. We could also identify a granular and a correlated regime in case of magnetized particles. The granular regime is characterized by quasi-continuous granular flows, while the correlated regime is characterized by long particle clusters falling at the system's boundary. The transition between the two regimes is not sharp. In simulations we found that the transition is at $f_{c} \approx 7$, while calculations based on stability criteria indicate a transition at $f_{c} \approx 6$ for the investigated magnetic case.

In the granular regime the avalanche sizes are proportional to the corresponding average avalanche durations. According to this, there is a well defined average granular flow characterizing the avalanches. We found that this increases linearly with $f$. Analyzing the avalanche size distributions, we also found that there is a well-defined characteristic size in particle avalanches. Based on scaling properties of the avalanche size distributions, we argue that the characteristic avalanche size increases linearly with $f$. The characteristic average avalanche duration in leading order seems to be independent of $f$. This dependence is contained in higher order corrections which could not be captured by our simulations.

In the correlated regime the avalanche sizes are in lead- ing order proportional to the square of the corresponding average avalanche durations. This is explained by the free fall of long particle clusters. The avalanche durations are defined by the length of the falling particle clusters. The width of the falling clusters is small, and can take arbitrary values up to a well defined maximum. According to this the avalanche sizes are defined in leading order by the cluster lengths, but there are large fluctuations which are also proportional to the cluster lengths. The cluster length increases linearly with $f$. The avalanche duration distributions show evidence of a characteristic avalanche duration, indicating also a characteristic avalanche size. We could not identify this avalanche size explicitly because of the large fluctuations in the avalanche size distribution.

Our results regarding the avalanche size distributions in both granular and correlated regime are very close to the experimental results of Tegzes et al. [6] on wet granular materials. They could identify characteristic avalanche sizes in both regimes, and they state that the avalanche size distributions in correlated regime are broad and both small and large avalanches may occur. This seems to confirm our finding that in correlated regime there are large avalanche sizes fluctuations.

The results on avalanche sizes and durations may slightly depend on the chosen time scale on which the avalanches are observed, however we argue that much more coarser or finer time scale will both lead to nonphysical results, while small correction in the time scale will not lead to qualitative difference.

\section{ACKNOWLEDGMENTS}

This research was carried out within the framework of the "Center for Applied Mathematics and Computational Physics" of the BUTE, and it was supported by BMBF, grant HUN 02/011, and Hungarian Grant OTKA T035028.
[1] A. J. Forsyth, S. R. Hutton, M. J. Rhodes, and C. F. Osborne, Phys. Rev. E 63, 031302 (2001).

[2] S. Hutton, Ph.D. thesis, Monash University (2002).

[3] D. J. Hornbaker, I. Albert, A. L. Barabási, and P. Schiffer, Nature (London) 387, 765 (1997).

[4] R. Albert, I. Albert, D. Hornbaker, P. Schiffer, and A. L. Barabási, Phys. Rev. E 56, R6271 (1997).

[5] P. Tegzes, R. Albert, M. Paskvan, A. L. Barabási, T. Vicsek, and P. Schiffer, Phys. Rev. E 60, 5823 (1999).

[6] P. Tegzes, T. Vicsek, and P. Schiffer, Phys. Rev. E 67, 051303 (2003).

[7] M. A. S. Quintanilla, J. M. Valverde, A. Castellanos, and R. E. Viturro, Phys. Rev. Lett. 87, 194301 (2001).

[8] A. Samadani and A. Kudrolli, Phys. Rev. E 64, 051301
(2001).

[9] L. Szalmás, J. Kertész, and M. Zrínyi, (unpublished), L. Szalmás, Diploma Work (in Hungarian), BUTE (2000).

[10] P. A. Cundall and O. D. L. Strack, Geotechnique 29, 47 (1979).

[11] G. H. Ristow, in Annual Reviews of Computational Physics I, edited by D. Stauffer (World Scientific, Singapore, 1994).

[12] H. J. Herrmann and S. Luding, Continuum Mech. and Thermodyn. 10, 189 (1998).

[13] S. Luding, in The Physics of Granular Media, edited by H. Hinrichsen and D. E. Wolf (Wiley-VCH, 2004).

[14] S. Fazekas, J. Kertész, and D. E. Wolf, Phys. Rev. E 68, 041102 (2003). 
[15] E. Altshuler, C. M. O. Ramos, L. E. Flores, and C. Noda, Phys. Rev. Lett. 86, 5490 (2001).

[16] L. D. Landau and E. M. Lifshitz, Theory of Elasticity (Pergamon, New York, 1970), chap. 9, (2nd English ed.).

[17] G. Kuwabara and K. Kono, Jpn. J. Appl. Phys. 26, 1230 (1987).

[18] H. Edelsbrunner, Computer Science, Tech. Report UIUCDCS-R-92-1760, Univ. of Illinois, Urbana (1992).

[19] N. Akkiraju, H. Edelsbrunner, M. Facello, P. Fu, E. P. Mucke, and C. Varela, in Proc. Internat. Comput. Geom. Software Workshop (1995), pp. 63-66.

[20] http://www.cgal.org, Computational Geometry Algorithms Library (CGAL 2.4) (2003).

[21] C. M. Dury, G. H. Ristow, J. L. Moss, and M. Nakagawa, Phys. Rev. E 57, 4491 (1998).
[22] Y. C. Zhou, B. D. Wright, R. Y. Yang, B. H. Xu, and A. B. Yu, Physica A 269, 536 (1999).

[23] Y. C. Zhou, B. H. Xu, A. B. Yu, and P. Zull, Powder Technology 125, 45 (2002).

[24] J. Feder, Fractals 3, 431 (1995).

[25] V. Frette, K. Christensen, A. Malthe-Sorenssen, J. Feder, T. Jossang, and P. Meakin, Nature (London) 379, 49 (1996).

[26] R. M. Costello, K. L. Cruz, C. Egnatuk, D. T. Jacobs, M. C. Krivos, T. S. Louis, R. J. Urban, and H. Wagner, Physical Review E 67, 041304 (2003).

[27] http://maxwell.phy.bme.hu/ fazekas/magaval, Avalanche movies of magnetized particles (2004). 